Open Peer Review on Qeios

\title{
Prestenting and its role in the URS: a study on the advantages it could have in intracorporeal lithotripsy interventions
}

Piero Mannone ${ }^{1}$ Rosa Giaimo, Davide Baiamonte, Gianpaolo Di Gregorio, Gabriele Tulone, Pinelli Mirko, Nicola Pavan, Marco VELLA ${ }^{1}$, Alchiede Simonato ${ }^{1}$

1 University of Palermo

Funding: The author(s) received no specific funding for this work.

Potential competing interests: The author(s) declared that no potential competing interests exist.

\section{Abstract}

Ureteroscopy (URS) represents the most used procedure in the treatment of urolithiasis, conversely ureteral stenting before URS is still a topic of scientific discussion. The aim of our study is to evaluate the possible benefits and uses of pre-stenting and if it can be used in the everyday practice. In our retrospective observational study, we collected data of patients undergoing URS for ureteral and renal lithiasis from January 2018 to December 2020 at our Department. Patients were stratified by procedure (ureterolithotripsy or URSL vs retrograde intrarenal surgery or RIRS) and by pre-stenting. We collected the patients' clinical characteristics and the timing of pre-stenting insertion. As outcomes variables, we analyzed the stone free rate (SFR), operating times (OT) and short-term complications. 244 patients were included in the study, 84 had undergone pre-stenting with a mean of $44.3 \pm 42.7$ days before surgery, while 160 did not. Regarding OT there are no differences in URSL and in the RIRS of stones in the renal pelvis; considering the RIRS of caliceal stones it is observed how the OT are lower in prestenting patients undergoing RIRS of caliceal stones (mean $79.29 \pm 34.7$ vs $91.08 \pm 53.8$ mins, $p=0.1$ ) and in case of multiple caliceal stones (mean $83.24 \pm 31.2$ vs $103.53 \pm 48.6$ mins, $p=0.1$ ). No differences were reported in the SFR for every URSL subgroups while it is higher in patients undergoing RIRS with a prestenting ( $85 \%$ vs $75 \%, p=0.08$ ). Regarding the short term complications, in the pre-stenting group fewer cases of post-operative pain $(20.4 \%$ vs $39.6 \% p<0.02)$ and hematuria $(14.8 \%$ vs $28.3 \% p=0.08)$ were reported. Our study is in accordance with the indications of guidelines which advise against the routine use of pre-stenting before URS. RIRS deserve a separate evaluation and our study, although considering the routine use of pre-stenting unnecessary, recognize its usefulness in achieving SFR and reducing intraoperative complications.

\section{Introduction}

Ureteroscopy (URS) represents the most used procedure in the treatment of urolithiasis. Technical improvements (introduction of smaller flexible and semi-rigid ureteroscopes, improved deflection 
mechanism, enhanced optical quality and tools, possible introduction of disposables) have led to an increased use of URS for renal and ureteral stones in the last years. In this field, ureteral stenting before URS is a topic of scientific discussion. According to American Urological Association (AUA) notes [1], the European Association of Urology (EAU) guidelines state that routine preoperative stenting before URS is not necessary [2]. However, pre-stenting has been shown to facilitate URS (in particular for renal stones) [3]; furthermore an improvement of Stone Free Rates (SFRs) and a reduction of intra-operative complications has been observed in different studies [4]. In this study we compare the outcomes (SFRs, operative times, short-term post-operative complications and success on placement of Ureteral Access Sheath) of patients who underwent to URS for renal and ureteral stones, with or without the use of a preoperative stent. The aim of our study is to evaluate the possible benefits and try to understand if stenting before URS can be more useful in the treatment of renal or ureteral stones.

\section{Methods}

In a retrospective observational study, we collected data of patients undergoing URS for ureteral and renal lithiasis from January 2018 to December 2020 in the Section of Urology of "Policlinico Paolo Giaccone" in Palermo. All surgical operations were performed by experienced surgeons. After collecting clinical data regarding age, gender, $\mathrm{BMI}$ and history of previous upper tract endoscopic surgery, patients were divided into two groups according to the presence of a ureteral stent placed prior to the procedure. Considering patients undergoing pre-stenting, it was evaluated the time elapsed between the procedure and the surgical operation. Both groups were stratified according to the procedure patients underwent (ureterolithotripsy or URSL vs retrograde intrarenal surgery or RIRS) and the characteristics of the lithiasis: whether single or multiple and the ureteral (proximal vs distal) or renal (calyxes vs renal pelvis) site. For all surgical operations were collected the operating times (OT), the eventual intraprocedural use of a ureteral sheath (UAS) and, in case of its use, its calibre. After every procedure, peri- and post-operative information as stone free-rate (intended as the absence of residual lithiasis or residual lithiasis up to a maximum of 4 $\mathrm{mm}$ ) detected by endoscopic control or radioscopic, the possible onset of fever, haematuria or postoperative pain, the Clavien-Dindo Classification and the positivity of any urine cultures were reported. Data was analyzed by using SPSS Statistics for Windows, Version 23.0 (IBM SPSS Statistics for Windows, Version 23.0. Armonk, NY: IBM). Data were compared with media-standard deviation (SD) and with unpaired T-test. A value of $p<0.05$ was considered significant. Discrete data were analyzed with contingency analysis. A Chi $2<0.05$ was considered significant.

\section{Results}

244 patients were included in the study (140 men e 104 women). 84 patients had undergone pre-stenting for urolithiasis (mean time: 44.3 days, SD: 42.75). Regarding patients' clinical characteristics, no statistically significative differences were found between two groups for BMI (pre-stenting group 26,8 \pm $5,02$ vs not pre-stenting group $26,46 \pm 5,76, p=0,67)$ and for age $(54,2$ yo $\pm 14,6$ vs 52,8yo $\pm 13,69$, 
$p=0,46)$. It was observed a statistically significative difference between the two groups for previous history of endourological procedure (pre-stenting $93.9 \%$ vs not pre-stenting $17 \%, p<0,001$ ). Regarding the operating time for total procedures (RIRS, URSL or combined), it resulted increased in pre-stenting group (mean $71.8 \pm 37.6$ vs $58.5 \pm 36.9$ mins, $p<0.0093$ ) while, considering the single procedure, there are no differences in URSL (mean $51.59 \pm 26.38$ vs $45.1 \pm 24$ mins $p=0.34$ ) and in the RIRS of stones in the renal pelvis (mean $72.22 \pm 38.89$ vs $73.62 \pm 39.25$ mins $p=0.9$ ). As regards the RIRS of caliceal stones, although not reaching statistical significance, it is observed how the means of OT are lower in pre-stenting patients undergoing RIRS of caliceal stones (mean 79.29 vs 91.08 mins $p=0.1$ ) and in particular in case of multiple caliceal stones (mean 83.24 vs 103.53 mins $p=0.1$ ). Regarding the SFR, considering the total procedures and the URSL it was comparable, while it is higher in the RIRS in pre-stenting patients ( $85 \%$ vs $75 \%$, $\mathrm{p}=0.08$ ). It was not used ureteral sheath in URLS procedures in both groups, while for RIRS an ureteral sheath was used with the same calibre and the same success positioning rate obtained in both groups. As regards short term complications, it has been seen that in pre-stenting patients there are fewer cases of post-operative pain $(20.4 \%$ vs $39.6 \% \mathrm{p}<0.02)$ and hematuria $(14.8 \%$ vs $28.3 \% \mathrm{p}=0.08)$. Complications reported with Clavien-Dindo classification were comparable between the two groups. In the end, there was a statistically significative increase in urine cultures positive rate in pre-stenting group $(27.4 \%$ vs $10 \%$, $\mathrm{p}<0.0004)$.

Our study has limitations that may have influenced the reported results. In particular, the major limitation is represented by the retrospective nature of the research which did not allow to analyze certain outcomes and to calculate an adequate sample size. Another limitation is represented by the lack of standardization of pre-stenting times which are heterogeneous in our series as we have analyzed the cases of patients who have undergone stent placement in urgency. Finally, the outcome regarding the caliber of the UAS is affected by the use of the same ureteral sheath caliber in our operating unit. However, our study has the merit of having considered the procedures separately, taking into account the location of the lithiasis and the possible presence of multiple lithiasis, comparing the interventions of URSL and RIRS with a high number of patients. Future perspectives will be to carry out a prospective study in order to create a nomogram that, based on factors derived from imaging techniques and the patient's clinical history, can identify all cases in which the use of pre-stenting can improve outcomes. and ensure a more effective intervention.

\section{Conclusion}

The results of our study are in accordance with the indications of the American and European Guidelines, which advise against the routine use of pre-stenting before URS. Especially in URSL, our study highlights an unbalanced cost-benefit ratio that does not justify its routinary use. RIRS deserves a separate evaluation and our study consider feasible the use of pre-stenting strategy, in particular in the case of calyceal and multiple stones. Similar to EAU evidence which, although considering the routine use of pre-stenting unnecessary, recognizes its usefulness in achieving SFR and reducing intraoperative complications in 
selected cases. Further studies, with greater cohort of patients, will be needed to better understand which patients need more pre-stenting and its correct timing.

\section{Reference}

1) Assimos D, Krambeck A, Miller NL, et al. Surgical Management of Stones: American Urological Association/Endourological Society Guideline, PART I. J Urol. 2016;196(4):1153-1160. doi:10.1016/j.juro.2016.05.090

2) EAU Guidelines. Edn. presented at the EAU Annual Congress Milan 2021. ISBN 978-94-92671-13-4.

3) Smith D. Pre-stenting and the risk of postoperative sepsis: a shorter dwell time is better. BJU Int. 2017;120(1):7-8. doi:10.1111/bju.13876

4) Assimos D, Crisci A, Culkin D, et al. Preoperative JJ stent placement in ureteric and renal stone treatment: results from the Clinical Research Office of Endourological Society (CROES) ureteroscopy (URS) Global Study. BJU Int. 2016;117(4):648-654. doi:10.1111/bju.13250 